\title{
La construcción de objetos en la Ciencia de la Información: el caso del proceso organización del conocimiento
}

The construction of scientific objects in Information Science: the case of the knowledge organization process

\author{
Rodrigo RABELLO \\ Instituto Brasileño de Información en Ciencia y Tecnología (IBICT), Brasil \\ rabello@ibict.br/rdgrabello@yahoo.com.br
}

\begin{abstract}
Resumen
El presente artículo explora las posibilidades de construcción de objetos científicos que orientan la creación de disciplinas situadas bajo el espectro de la Ciencia de la Información ( $\mathrm{Cl})$. Por lo tanto, presenta un modelo teórico que considera ilustrativamente la construcción del objeto organización en lo que se refiere al proceso de constitución disciplinar de la Organización del Conocimiento (OC). Considerando los presupuestos bachelarianos sobre la construcción de objetos científicos se presenta una retrospectiva histórica de la OC cuya delimitación disciplinaria se orienta no solamente por su objeto, sino que también por su función social. Se argumenta que el ejemplo del proceso organización —-tomado como objetoabre margen para la reflexión sobre la proposición de otras disciplinas que componen el cuadro teórico de la Cl.
\end{abstract}

Palabras clave: Construcción de objetos científicos. Organización del Conocimiento. Epistemología. Ciencia de la Información.

\section{Introducción}

En la formulación de una teoría, los conceptos se vuelven relevantes para sustentar explicaciones, de forma lógica y coherente, sobre un determinado objeto a partir de los resultados obtenidos con la aplicación de uno o más métodos particulares. Dependiendo del valor dado a los conceptos en un campo disciplinar, el entendimiento del fenómeno principal $-u$ objeto-que la disciplina se propone estudiar puede, en términos epistémicos, quedar desvirtuado. Una alternativa a la situación descrita puede ser pensada a partir de la premisa de que la comprensión del proceso de construcción de objetos corroboraría para situarlos -contextualizarlos disciplinariamente- auxiliando al entendimiento de la delimitación de la propia disciplina.

En esa dirección, el presente artículo busca apoyos teóricos para comprender la interdependiente relación entre la construcción del objeto,

\begin{abstract}
This paper explores the possibilities of constructing scientific objects that guide the creation of disciplines situated under the spectra of Information Science (IS). For such, a theoretical model considering illustratively the construction of the organization object is regarding the disciplinary constitution of Knowledge Organization is presented (KO). Considering bachelarian assumptions about the construction of scientific objects, herein is presented a historical retrospect of $\mathrm{KO}$ whose disciplinary delimitation is oriented not only by its object, but also by its social function. It is argued here that the example of the organization process taken as an object- gives way for the reflection on the proposition of other disciplines that compound the theoretical framework of IS.
\end{abstract}

Keywords: Construction of scientific objects. Knowledge Organization. Epistemology. Information Science.

los conceptos auxiliares y la conformación de la disciplina científica. El punto de partida es el espectro disciplinar de la Ciencia de la Información $(\mathrm{Cl})$ o, en la expresión de Esteban Navarro (1995), de las ciencias documentales donde el proceso organización es considerado como objeto de estudio que ha llevado a la construcción disciplinar de la Organización del Conocimiento (OC).

El modelo teórico presentado a continuación tendrá como aporte introductorio los presupuestos bachelarianos sobre la construcción de objetos científicos. Esa discusión abrirá camino para situar al proceso organización como objeto, así como también presentará una retrospectiva histórica de la OC que no prescindirá de comparar su delimitación disciplinaria con su función social. 


\section{La construcción de objetos científicos}

La ciencia moderna emergió a partir del momento en que el método pasó a tener mayor relevancia para la producción de conocimientos, en particular, con la creación de disciplinas como la Geometría y la Aritmética. La búsqueda de la sustancialidad de las cosas, por medio de la forma y de la materia, fue una orientación que configuró y justificó los primeros saberes positivos. De acuerdo con Vullemin (1987, p. 117), ese presupuesto abrió un espacio para el estatuto metodológico en un lugar donde predominaba el ontológico, o sea, el estatuto filosófico dedicado al estudio del ser. Así, en la ciencia moderna pasó a ser considerada la capacidad metódica de comprender la substancia real $-u$ objeto- pausible de observación.

Cuestiones sobre el método científico fueron abordadas por el epistemólogo francés Gaston Bachelard (1884-1962), cuya actuación pasó por la Filosofía y por la Poética. De la obra del autor, se presenta, en este artículo, su reflexión en el campo filosófico sobre Epistemología e Historia de las Ciencias, enfatizando, específicamente, así como lo ha hecho Melo (2006), el análisis de Bachelard acerca del potencial metodológico que sintetiza la noción de "construcción del objeto científico".

Tomando como punto de partida la innovación científica conquistada por Albert Einstein en el siglo $\mathrm{XX}$, en su teoría de la relatividad, Bachelard discurrió sobre la historicidad de la epistemología, así como sobre la relatividad del objeto. En ese cuadro, la relatividad de la ciencia, para Bachelard (1972, p. 27), partió de la constatación de que "[...] el espíritu científico contemporáneo no podía ser colocado en continuidad con el simple buen sentido." El momento fue propicio para una ruptura con el sentido común, constantemente empleado en las formulaciones empiristas, movidas por la percepción sensorial y por la apariencia inmediata de los fenómenos observados.

Al ceder lugar al racionalismo - para formar el "nuevo espíritu científico" - la ciencia pasaría a orientarse por el primado de la realización sobre la realidad. En otras palabras, el vacío teórico, presupuesto anteriormente en las experiencias directas, pasaría a primar por la valorización de la teoría que ilustra el método durante la aproximación del científico al objeto estudiado. La mediaticidad del método científico por la razón pasó a adquirir valor estratégico en el trayecto de lo racional a lo real. Se admite, dentro de esos términos, la superación del empirismo por el racionalismo.
Al estudiar el progreso de las condiciones internas, psicológicas, del conocimiento científico, Bachelard (1996a) evidenció la existencia de "obstáculos epistemológicos". Con base en el universo psicoanalítico, el autor avanza la hipótesis de que existe una vía psicológica del pensamiento científico, en cuyas condiciones normales "[...] el acto de conocer se da contra un conocimiento anterior, destruyendo conocimientos mal establecidos, superando lo que, en el propio espíritu, es obstáculo a la espiritualización" (Bachelard, 1996a, p. 17).

A partir de la noción de que la actuación del científico es responsable de la superación de tales obstáculos - sea durante el proceso de investigación o en la obtención de los resultados-, dos obstáculos, como bien lo señaló Melo (2006), adquieren real destaque: el "obstáculo de la realidad" y el "obstáculo del sentido común".

El "obstáculo de la realidad" se refiere a que, sobre todo en el ámbito de las Ciencias Sociales, existe el riesgo de que el científico sea traicionado por lo que es aparentemente visible en su relación empírica. De forma similar, el "obstáculo del sentido común" se da frente a la dificultad del científico social de librarse, al analizar el objeto, de su conocimiento común, opiniones, pre-conceptos, etc. (Bachelard, 1996a, p.18).

En esa dirección, es posible deducir que el objeto científico no representa simplemente un continuum de la naturaleza. Es el científico que lo construye cuando busca aproximarse mediatamente del objeto creado con el auxilio de una teoría que, al mismo tiempo, ilustra y es ilustrada por un método.

Con el racionalismo, la realidad representada por el científico adquiere la condición de parcialidad en lugar de la totalidad antes imaginada por la observación directa. En efecto, el espacio - o el fenómeno- con el cual el científico entra en contacto es, ante todo, fruto de una representación.

En esos términos, Bachelard (1996b, p. 101) defiende que el realismo científico también debe ser superado en condenación, por ejemplo, a la doctrina cartesiana de las naturalezas simples y absolutas. Eso es porque Decartes acreditaba en la "existencia de elementos absolutos en el mundo objetivo" y pensaba que esos elementos eran "conocidos en su totalidad" por medio de la experiencia directa.

En el racionalismo, la multiplicidad de métodos es un aspecto importante para la propia construcción de los objetos. Considerando que la particularidad del objeto influye en la elección 
del método, este, al ser aplicado, modifica y determina al objeto, pudiendo igualmente modificarse con los resultados obtenidos con la demonstración. En esos términos, el método deja de tener una connotación de instrumento estático. La rigidez metodológica no es apropiada para el proceso creativo del científico que construye y reinventa su objeto de estudio. Las experiencias nuevas tienden a modificar el pensamiento científico en la medida en que, segun Bachelard (1996a, p. 97), un "[...] discurso sobre el método científico será siempre un discurso de circunstancia, no describirá una constitución definitiva del espíritu científico."

La creación de disciplinas científicas, a la luz del contexto explicitado por Bachelard, puede ser pensada a partir de la actividad de un colectivo particular de científicos -en la expresión utilizada por Kuhn (2005), una comunidad científica- actuando en la construcción y delimitación de objetos con vista a su control mediante la aportación de métodos y teorías. De la disciplina se deducen las ideas de delimitación, conformación, clausura y asentamiento de un conjunto de conocimientos presentados con una figuración racional específica para la formación de una unidad auxiliadora del proceso de estudio investigación, enseñanza y aprendizaje.

Esa concepción lato sensu de disciplina como rama del saber — ciencia- es pensada en sus bases operacionales por medio del proceso de compartimentalización del conocimiento científico en el ámbito académico-profesional y administrativo. La institucionalización de la ciencia en escuelas y universidades ocurre mediante la estructuración de matrices (o componentes) curriculares de cursos cuyas materias son elaboradas y organizadas siguiendo la lógica disciplinar para viabilizar la investigación, la enseñanza y el aprendizaje (Pombo, 2003). Otro aspecto está relacionado con la clasificación de áreas por órganos del gobierno para regulación, evaluación o financiación de la investigación y de la enseñanza de las especialidades (González de Gómez, 2003). En síntesis, el término disciplina, stricto sensu, puede connotar: a) las partes o materias que componen las estructuras curriculares, o b) el resultado de las clasificaciones y representaciones de áreas, para asuntos administrativos. Así, es posible decir que el sentido lato de la designación, en el contexto epistemológico, se relaciona de forma interdependiente de su sentido stricto en el ámbito institucional.

$\mathrm{Si}$, por un lado, el término ciencia se refiere al conocimiento, o epistéme, logrado por medio de la aplicación de algún criterio metódico y organizado de tal forma que pueda ser disciplinado; por otro, el término disciplina remite al resultado del esfuerzo de delimitación de la ciencia como una rama del saber. En ese sentido, ambos términos se presentan como sinónimos, aunque puedan remitir también a "dos caras de una misma moneda", cada cual con particularidades que pueden ser percibidas en una dinámica caracterizada por la interdependencia de las "caras" para formar la unidad.

La conformación disciplinar ocurre en el proceso de especialización del conocimiento con el cual la comunidad científica delimita objetos con vista a su control por medio de la provisión de métodos y teorías. Así, la disciplina es la forma por la cual la ciencia encuentra su "puerto seguro" - aunque provisionalmente - para la búsqueda del conocimiento. El carácter provisional de la disciplina - perceptible en la coherencia lógica que sustenta la concepción de revolución científica de Kuhn- sugiere la efemeridad de elementos de su propia conformación.

Para ilustrar tal transitoriedad, Freire-Maia (1997, p. 17-18) acuño las expresiones "cienciadisciplina" y "ciencia-proceso" que auxilian la visualización de las "dos caras de la misma moneda" mencionada. Se presentan, a continuación, ambos conceptos:

- "ciencia-disciplina": expresión referente al proceso de constitución de una disciplina a partir de la presentación formal de un conjunto de descripciones, interpretaciones, leyes, teorías, modelos, etc., que apuntan al conocimiento de una parcela de la realidad y que son el resultado de la aplicación de una metodología científica especial.

- "ciencia-proceso": designación que considera el conocimiento científico "en vías de ser construido", en continua evaluación, cuyas afirmaciones positivas están a disposición del ejercicio del cuestionamiento, que podrá llevar a la su refutación. La dinámica de la "ciencia-proceso" puede estremecer las bases de la "ciencia-disciplina" pues en cuanto la "[...] ciencia-disciplina [pueda] parecer una edificación terminada, irretocable, llena de verdades (las 'verdades científicas')", la "ciencia-proceso", al contrario, "[...] revela que se trata de algo en permanente elaboración, ampliación y revisión."

Luego, la idea de univocidad semántica entre disciplina y ciencia ocurre, en parte, por la posibilidad de incidencia de dos movimientos complementares que, en términos ideales kuhnianos, actuarían en: a) la delimitación de positividades en dirección a la creación de la ciencia normal —búsqueda por validación de la perma- 
nencia, y b) en el cuestionamiento de los conocimientos consolidados, acumulados, disciplinados, mediante formulación de enigmas y anomalías hacia la ciencia extraordinaria y, en fin, a una nueva ciencia - búsqueda por validación de cambios, de transformación.

La posible simultaneidad de los movimientos citados puede ser ilustrada todavía por la búsqueda del conocimiento científico con dos finalidades: a) la resolución de problemas empíricos o pasibles de observación, medición y/o control mediante métodos cuantitativos, sumándose a la posibilidad de su interpretación, por medio de métodos cualitativos, y b) la sustentación de investigación pura, es decir, básica o fundamental, en búsqueda de nuevos conocimientos sin interesarse necesariamente por realizar aplicaciones pragmáticas.

En las próximas secciones se buscará contemplar algunas explicaciones formuladas por teóricos de la $\mathrm{Cl}$ que llevaran en consideración ambos movimientos — pensando de modo simultáneo disciplina/ciencia como cierre y apertura (1) - para la obtención de conocimiento por medio de la delimitación de objetos de estudio.

\section{El proceso organización como objeto}

Dentro del espectro de procesos de interés del estudio de la $\mathrm{Cl}$, destacan la producción, organización, recuperación, diseminación, acceso y uso de la información. Se puede decir que el fenómeno información es investigado por la $\mathrm{Cl}$ tanto en el trayecto de la producción y organización para el uso —diseminación-, como en el trayecto que considera el uso, bajo la presuposición del sistema organizado, para llegar a la información registrada y su recuperación. En ambos trayectos, las tecnologías de información se presentan como importantes instrumentos para la automación del sistema informativodocumental y para la optimización de productos y servicios de información mediante las posibilidades interactivas proporcionadas por el ambiente virtual de la Web.

Cabe destacar que tales trayectos ilustran, en cada proceso, los objetos de estudio que propician delimitar las principales disciplinas observadas bajo el espectro de la $\mathrm{Cl}$. Con la excepción del proceso producción de la información, que está presupuesto en los demás, los otros procesos han orientado la construcción de disciplinas. Por ejemplo, el proceso diseminación es objeto de la Diseminación de la Información; el proceso uso, que remite a quien usa, es objeto de la disciplina Estudio de Usuarios; el proceso recuperación es objeto de la Recuperación de la Información; y, por fin, el proceso organi- zación, que es objeto de la disciplina Organización de la Información.

Es importante mencionar que existen otros procesos relacionados a los citados que amplían la gama de disciplinas. Por ejemplo, a partir del proceso organización otros procesos/objetos como análisis (para Análisis Documental), indexación, clasificación, elaboración de resúmenes, catalogación, etc.- se desdoblan, acarreando la construcción de un cuadro teóricometodológico propio (Anderson, 1996). Cada proceso está relacionado a una actividad técnico-documental particular —con objetivos, funciones, procedimientos, etc. Esa condición contribuye a que el proceso sea el referencial director en la construcción y en la delimitación de la disciplina en la cual actuará como el principal objeto de estudio.

Considerando el proceso organización -en la condición de una actividad intelectual de la $\mathrm{Cl}$ de reflexión teórica que incide directamente en la condición de una etapa mediadora entre la producción y el uso del conocimiento - se objetiva presentar, en la próxima sección, algunos apuntes sobre aspectos concernientes a la construcción disciplinar del área de organización, justificándola, sobre todo, por su función social de transmisión de conocimiento.

Para diferenciar de la concepción de Rabello y Guimarães (2006), la perspectiva presentada considerará el proceso como objeto definidor de la disciplina, dislocando para un segundo plano otros supuestos objetos, como, por ejemplo, conocimiento, información y documento. Se parte de la hipótesis de que aunque tales conceptos ocupen un lugar secundario cuando se les compara con el objeto organización, ellos no pierden importancia en el cuadro teórico de la disciplina, ya que ayudan a comprender diferentes fenómenos relacionados con el proceso, ilustrando, principalmente, su dimensión social.

Eso corrobora la característica apuntada por Bachelard (1996a) de que la ciencia racionalista no trabaja con objetos en sí, sino con las relaciones que los determinan. En ese sentido, el objeto científico organización es construido en su necesaria relación con otros objetos y conceptos.

A partir del citado presupuesto bachelariano, se considerará, en este texto, la expresión Organización del Conocimiento enfatizando más el proceso que la noción abstracta (conocimiento o información) o concreta (documento) a la que se propone incidir, aunque tales conceptos presenten una posición relevante para ayudar a situar el propósito del objeto (proceso) estudiado. 


\section{Aspectos históricos de la organización del conocimiento}

Con el desarrollo de la escritura y de sus formas técnicas de producción y reproducción documental -en particular, después del invento de la tipografía gutenberguiana - fue posible ampliar las formas de transmisión del conocimiento acumulado a las generaciones venideras. El advenimiento de la imprenta se presentó como el "divisor de aguas" para una nueva relación entre hombre y conocimiento.

El cambio cualitativo en la relación entre el hombre y el conocimiento fue estudiado por Burke (2003) en su historia social del conocimiento. El historiador investigó el inicio del periodo moderno — de 1450 a 1750 -y constató una carencia de estudios sobre la temática en ese intervalo de tiempo. Ese periodo, en Europa, estuvo marcado por las revoluciones culturales, científicas y filosóficas, y por el surgimiento de la imprenta tipográfica y por la publicación de la primera enciclopedia. El desarrollo de las técnicas de impresión fue determinante, pues estimuló la convergencia dinámica de diversos tipos de conocimientos históricamente imprescindibles para el avance en la producción, expresión, expansión, aprehensión y diseminación del conocimiento para una infinidad de mudanzas significativas en el plano sociocultural. El autor menciona, por ejemplo, la ampliación de la función de la biblioteca, que dejó de ser solamente un espacio del silencio y de la lectura, y pasó a ser, también, un lugar de sociabilización de ideas y de intercambio de información, como ya ocurría en otros espacios públicos. Finalmente, la creación de la enciclopedia revolucionó las posibilidades de investigación como una forma innovadora de "recuperación de la información", influyendo en la creación de una nueva etapa del "consumo de cultura" y del "comercio del conocimiento". El desarrollo de la enciclopedia trajo, también, los principios de organización del conocimiento que influyeron, más tarde, en la creación de los primeros sistemas de clasificación documental (siglos XIX y XX).

Concomitantemente, el desarrollo de la "industria editorial" y del consecuente mejoramiento de la técnica de producción del libro - popularizado por su practicidad, manoseo, etc., y por la cualidad de impresión - transformó este objeto que hasta entonces había sido el más importante medio de registro y de divulgación del conocimiento (2). Su importancia aún perdura, como puede ser visto en su convivencia con otros soportes informacionales que también están destacando, como los electrónicos.
En el siglo XIX, el desarrollo de la industria citada permitió que se produjera la producción documental en gran escala. El aumento de la producción de documentos llevó a la dificultad de organizarlos, causando un problema a la sociedad moderna en lo que se refiere a la recuperación del conocimiento científico acumulado para la generación de nuevos conocimientos (Woledge, 1983).

Ese contexto fue propicio para la creación de iniciativas orientadas al tratamiento documental, buscando la organización de los documentos para su recuperación y uso. Ese periodo de mejoramiento técnico-documental tiene como pionero al bibliotecólogo estadounidense Melvil Dewey, creador del sistema de Clasificación Decimal de Dewey - CDD - con el objetivo de facilitar la organización del material bibliográfico en las bibliotecas.

Paralelamente a la preocupación por los libros en las bibliotecas, el mejoramiento técnicodocumental necesitó sintonizarse con las demandas de la especialización del conocimiento, la cual encontró en la revista científica uno de los principales medios de divulgación. A partir de ese momento, las bibliotecas no deberían (Fayet-Scribe, 2001, p. 14)

[...] ser apenas un simple almacen de libros, donde estos se acumulan sin crear los medios de acceso necesarios a sus contenidos. En esta perspectiva, el papel de la revista científica es central porque cuestiona el papel de las unidades documentales: la unidad no es ya el libro, sino el artículo en el interior del documento.

En el ambiente de la ciencia, la revista afectó a la noción de libro, que era concebido por la bibliotecología "clásica" como el objeto que traía una unidad del conocimiento, un "contenidouno". La noción de periódico, en su pluralidad de asuntos separados en artículos, mostró que el libro también puede ser observado y tratado documentalmente en su extensión considerando la diversidad de asuntos que contiene.

Con el aumento de la importancia de los periódicos científicos crecieron las iniciativas de elaboración de bibliografías especializadas. Tales iniciativas acompañaron el amplio desarrollo de las ciencias, ganando proporciones mayores en un momento en el que tales bibliografías eran elaboradas con el patrocinio de asociaciones de investigadores.

Fue en ese contexto en el que Paul Otlet inició sus primeras reflexiones sobre la práctica de la organización bibliográfica a finales del siglo XIX. Al catalizar las demandas de conocimiento científico de su época - además del trabajo de articulación política, por medio de la creación de 
asociaciones e instituciones dirigidas al tratamiento documental- el autor belga elaboró varios textos publicados en periódicos y presentados en eventos, que dieron como resultado la sistematización teórica, en 1934, del Traité de Documentation, obra que contiene los fundamentos disciplinares de la Documentación.

Después de fundar el Taller Internacional de Bibliografía, en 1892, y el Instituto Internacional de Bibliografía, en 1895, Otlet creó el Repertorio Bibliográfico Universal que había de ser un instrumento que permitiría el acceso al conocimiento registrado producido en ámbito internacional. Según Otlet (1934, p. 381), el Repertorio Bibliográfico Universal fue concebido como un

[...] catálogo en el que se registra integralmente y se clasifica toda la producción intelectual, con la forma de un inventario (lo que lo hace mucho más accesible para todas las personas y para todas las finalidades).

La organización del conocimiento registrado sería posible mediante su clasificación previa por medio de Clasificación Decimal Universal CDU - creada a partir de la CDD, de Dewey. Con ese sistema, hipotéticamente sería posible clasificar todos los "[...] conocimientos [que] aparecen representados en todo su ciclo: la enciclopedia del saber". (Otlet, 1934, p.381).

Con la CDU, no solamente los libros sino cualquier documento podría ser clasificado. La CDU fundamentó la necesidad de considerar al libro como un conjunto de asuntos posibles de ser desmembrados para fines de organización y recuperación de contenido. También amplía Otlet el concepto de documento, pues, a partir de entonces, fueron tomados en cuenta los contenidos documentales expresados en diferentes soportes y formatos (fotografías, mapas, etc.). Con Otlet, el libro dejó de tener una noción restrictiva y fue retomada la amplia concepción de biblos de la Antigüedad y de la Edad Media, en la que se consideraban todos los soportes artificiales del conocimiento (Rendón Rojas, 2005; Rabello, 2009, 2011).

Además de la CDD y de la CDU, fueron creados otros sistemas, entre los cuales merece destacarse la Clasificación colonada (Colon Classification), desarrollada por Shiyali R. Ranganathan, en 1933. La clasificación de Ranganathan fue innovadora por presentar un esquema de clasificación basado en el denominado análisis de facetas.

El objetivo de la clasificación facetada consiste en acompañar los cambios y la evolución del conocimiento humano, mediante un método analítico-sintético, para cumplir dos funciones: 1) permitir la organización de los documentos en los estantes; y 2) representar el conocimiento registrado en diferentes áreas. El proceso de clasificación facetada se constituye en tres etapas: 1) análisis temático o conceptual, plano de las ideas; 2) traducción, plano verbal; y 3) construcción de notación, plano notacional, simbólico (Campos, 2001).

La teoría de la clasificación de Ranganathan continua siendo de gran importancia para los estudios sobre organización del conocimiento, ganando mayor dimensión, notoriamente después de su profundización teórica por autores como Coates, Farradane, Foskett y Vickery. El análisis de facetas (Fujita, 2001, pp. 30-31)

[...] posibilitó que los asuntos pudieran ser analizados de diferentes formas conforme al contexto ambiental o temático, trayendo a la clasificación y a la Bibliotecología una condición teórica que exigió una relación con otras áreas del conocimiento implicadas con el objetivo de la OC, para profundizar en sus aportaciones teórico-metodológicas. Eso propició la interdisciplinaridad, principalmente, con la Lingüística aplicada y la Terminología, la Informática y la Inteligencia Artificial, la Psicología y los estudios psicolingüísticos en la perspectiva cognitiva, la Filosofía y la Semiótica.

El estudio del análisis de facetas fue de la misma forma importante para el establecimiento de vocabularios controlados y para la elaboración de tesauros. El tesauro es un tipo de lenguaje documental (3) que tiene como característica conceptual el partir de una idea en dirección a las palabras. Los diccionarios siguen un camino opuesto. En la estructura del tesauro, los términos son relacionados (genéricamente) para cubrir un dominio específico de conocimiento. Ese instrumento de indización tiene la función de ser un dispositivo de control de vocabulario, es decir, de traducir contenidos de acuerdo con el lenguaje del sistema de recuperación de la información.

A partir del final de la II Guerra Mundial se intensificaron los trabajos sobre tesauros. Se observan, por ejemplo, las iniciativas realizadas en la década de 1960 por la Armed Services Technical Information Agency (ASTIA) y por el Engineers Joint Council (EJC), en los estados Unidos. Tales trabajos motivaron la creación posterior, en Alemania, del Comité de Investigación sobre Tesauros. Ese Comité estableció directrices de cómo los tesauros debían ser construidos, mantenidos y utilizados, y ubicó la creación de otros tesauros (y libros sobre el tema), conforme a normas nacionales e internacionales (Dahlberg, 1995, p. 8).

Por lo tanto, los tesauros fueron una alternativa a la representación sistemática de documentos por medio de los sistemas de clasificación. Se 
presentaron como un instrumento importante para la representación y organización del conocimiento, aprovechándose, en un primer momento, de la tradición de la categorización alfabética de materias de Cutter, sin despreciar las jerarquías clasificatorias.

Conforme recuerda Ingetraut Dahlberg, la propia autora reconoció, junto con sus colegas del Comité Alemán de Tesauros, la existencia de una falla en la concepción de los tesauros elaborados po entonces, pues no se tomaban en consideración los avances ocurridos recientemente y las lenguas nativas, empobreciendo, de esa forma, a los sistemas de vocabulario controlado (Dahlberg, 1995).

Tal constatación se dio a partir de los estudios de Jean Aitchison que innovó al producir, en 1968, el primer Tesauro Facetado para la Compañía Eléctrica Inglesa, tomando como referencia el Colon Classification de Ranganathan y las experiencias adquiridas en el Grupo Inglés de Investigación Clasificatoria. El gran avance parte del diagnóstico (Dahlberg, 1995, p. 9) de que en el

[...] abordaje inductivo de la producción de tesauros - comenzando por términos y desarrollándose desde esa base, el concepto se agrupa con términos relacionados más incluyentes y más restrictivos, así como con sus equivalentes lingüísticos, en la formación de sinónimos y cuasi-sinónimos- fue complementado con el análisis deductivo partiendo de las disciplinas y su posible estructura conceptual.

A partir de ese presupuesto, Dahlberg crea, en la década de 1970, aquel que sería su marco teórico para la construcción de tesauros -la teoría del concepto. Esa teoría fue igualmente creada tomando como base la teoría de la clasificación. La autora buscó en la concepción de faceta la base para la creación de lenguajes documentales, como los tesauros terminológicos. La teoría del concepto parte de un esfuerzo teórico de definir término y concepto y de defender el empleo de categorías para la organización de conceptos. Conforme señala Campos (2001), la teoría del concepto tiene como objeto evaluar la consistencia de la relación entre lenguaje-pensamiento-realidad mediante la incorporación del referente en la tríada formada por el referente (objeto), característica (predicaciones) y término (representación).

Esa reciente perspectiva incentivó a Dahlberg y su grupo a dejar el Comité de Tesauro, quedando a cargo de la Sociedad Alemana de Documentación, y a fundar, en 1977, la Sociedad para la Clasificación. La Sociedad pasó a considerar como instrumental, en definitivo, la teoría de facetas y el abordaje taxonómico para la ordenación de objetos. Después de varias reuniones en los fórums de debate de la Sociedad para la Clasificación, nuevamente aconteció una disidencia por entenderse que la clasificación era solamente un proceso de la organización del conocimiento. De forma más amplia, se pasó a comprender organización del conocimiento como toda la estructura por la cual el "[...] conocimiento puede ser entendido, organizado, descrito y representado de tal forma que pueda ser accecido de modo apropiado y estar disponible a cualquiera que lo busque" (Dahlberg, 1995, p. 9-10). Con el objetivo de divulgar esas ideas a otros países, en 1989 fue creada la International Society for Knowledge Organization -ISKO.

La ISKO paso a ser, a partir de entonces, una importante institución para el desarrollo teórico y la construcción disciplinar de la OC. Para eso, los teóricos de la ISKO se han ubicado dentro de un abordaje interdisciplinar preocupado por las cuestiones tangentes al tratamiento documental y a la diseminación del conocimiento. Como consecuencia, los fórums internacionales de debate de la ISKO se convirtieron en referencia en el área. Además de la propia Dahlberg (creadora de la ISKO), en el contexto de la IS$\mathrm{KO}$ internacional se destacan investigadores como Hjørland, Albrechtsen, Ingwersen, Poli, Olhy, Beghtol, Mc Ilwaine, Janecke, Green, entre otros; en los capítulos de la ISKO del eje Ibero-Americano, se destacan López Huertas, García Marco, Esteban Navarro, Barité, Guimarães, entre otros.

\section{Aspectos disciplinares y sociales de la Organización del Conocimiento}

El área de organización se destaca en la $\mathrm{Cl}$ por traer reflexiones teóricas acerca de las concepciones y métodos que caracterizan la actividad mediadora entre la producción y el uso de la información. El empleo de las expresiones Organización del Conocimiento y Organización de la Información, comúnmente observadas en la literatura del área, sugiere cierto impás con relación al nombre usado para designar la disciplina. Además, a las dos expresiones se les ha acoplado frecuentemente la palabra representación, la cual remite a los productos documentales demandados por el proceso de organización.

Cabe afirmar que la expresión Organización del Conocimiento (Knowledge Organization) sobresale, ya sea porque ha sido oficialmente adoptada por la ISKO, o porque considera al termino conocimiento un ente ideal, que puede abarcar informaciones contextualizadas a) por su pro- 
ducción (acto intelectual y racional), b) materialización (registro en un soporte), y c) uso atribución de valor documental al objeto seguido de su lectura, aprehensión, etc.

El primer investigador que ha usado la expresión Organización del Conocimiento fue Henry Evelyn Bliss, concibiéndola como campo disciplinar autónomo. De la obra de Bliss se destacan sus libros The Organization of Knowledge and the System of Sciences, de 1929, y Organization of Knowledge in Libraries and the Subject Approach to Books, de 1933, que son considerados una referencia para el área (Dahlberg, 1995, p. 10). En esa dirección, se registran, también, las tesis académicas de Dagobert Soergel, que en 1971 defendió la relación de la organización del conocimiento con la documentación, y de Ingetraut Dahlberg, que en 1973 se preocupó de las bases teóricas del área.

Como se mencionó anteriormente, el origen de las actividades de organización del conocimiento aconteció con las prácticas de almacenamiento y recuperación de documentos "[...] en el transcurso de un proceso de perfeccionamiento de la sistematización del conocimiento humano." (Fujita, 2001, p. 29). Tales actividades se alinearon, en un principio, con el campo bibliotecológico, en particular, con la creación de "[...] sistemas de clasificación como instrumentos de organización temática de documentos para su almacenamiento" (Fujita, 2001, p. 29).

Tales actividades culminaron en la propuesta disciplinar, en el siglo $X X$, de la $O C$. Según apunta Barité (2001, p. 40), esta es una disciplina aplicada que se preocupa en producir fundamentos teóricos orientados a abarcar el continente conceptual adecuado a las diversas prácticas sociales en lo que se refiere al tratamiento temático de la información y, de modo menos específico, pero no menos importante, a los estudios de gestión del uso social de la información.

La OC ha contemplado, de forma integrada, los fenómenos y las aplicaciones relativas a la estructura, disposición, acceso y difusión del conocimiento socializado, o sea, registrado. La disciplina se preocupa, por lo tanto, con el acceso al referido conocimiento, operando con instrumentos de tratamiento, gestión y uso de la información.

De acuerdo con Hjørland (2003), el concepto de $\mathrm{OC}$, pensado en el campo de la $\mathrm{Cl}$, es amplio. Para ese autor, la disciplina puede ser aprendida en su variedad de sentidos relacionados con las actividades intelectuales y las técnicas de organización de la información que proporcionan fundamentos teóricos para la elaboración de registros bibliográficos. Tales registros se presentan como herramientas relevantes que optimizan la organización de la información en sistemas informativo-documentales -bibliotecas, bases de datos en línea, etc. En ese proceso, los principales actores son los productores de conocimiento (autores, editores, etc.), los intermediarios (profesionales de la información) y los usuarios reales interesados en el acceso y uso de la información organizada.

Además de la participación de esos actores, es importante destacar que la OC recibe la influencia de dos niveles de organización que pueden interrelacionarse:

- Organización intelectual del conocimiento u organización cognitiva: Consiste en la organización del conocimiento en conceptos, sistemas conceptuales y teorías. Por ejemplo, existe la tabla periódica de Química, la taxonomía zoológica en la Biología, etc.

- Organización social del conocimiento: Consiste en la organización en profesiones, acuerdos, disciplinas, así como en la organización del conocimiento prevista por convenciones sociales.

El objetivo da OC, según Jaenecke (1994), es "ordenar y suplir el conocimiento." En ese caso, se infiere la doble función de la disciplina:

- Función teórico-práctica, relacionada con la producción de conocimiento para pensar el propio proceso de organización;

- Función social, que impele la función teórico-práctica al objetivo último de la disciplina que es ordenar y contextualizar la masa de conocimiento registrado existente para que esta pueda suplir la demanda social de conocimiento.

Tales funciones pueden ser observadas a partir de la premisa de que el conocimiento se realiza a partir de la información. El registro del conocimiento ocurre en soportes que posteriormente tendrán valor documental, permitiendo, así, su socialización (4). La organización del contenido documental es manejada mediante sistemas de conceptos -con fines científicos, funcionales o de documentación-, siendo pautada por un tono artificial, provisorio y funcional, con la finalidad de promover su mejor aprovechamiento social. Al socializarse, el conocimiento nuevamente se transforma en información, poseyendo estructura y procesos de comunicación abiertos, capaces de transformarse ilimitadamente. A partir de esa dinámica es posible visualizar el proceso cíclico de producción de conocimiento, 
organización, recuperación, acceso, uso y producción de nuevo conocimiento (5).

El trayecto citado fue abordado por Barité (2001, p. 42-53) cuando relacionó el proceso de organización a la dinámica entre conocimiento e información que determina y posibilita su socialización por medio del documento. Para ese autor, el conocimiento entendido en la OC es aquel pausible de registro en documentos; los cuales serán susceptibles, también, de formar un conjunto organizado de información socialmente disponibles y que admiten usos indiscriminados.

Es importante esclarecer que en el contexto de la OC la noción de registro del conocimiento y de la información remite a dos formas documentales distintas, aunque mutuamente necesarias para la realización del proceso de organización: a) documento -insumo básico cuyo contenido será representado y ordenado por medio de instrumentos creados bajo la orientación de lenguajes documentales (tesauros, sistemas de clasificación, listas de encabezamiento de materia, etc.), también subentendidas como lenguajes artificiales o lenguajes del sistema de recuperación de la información-; y b) información o registro documental — producto documental, descodificación del contenido del documento (índices, notaciones de materia, etc.), forma que el contenido del documento asumirá al ser representado conforme a la orientación de un lenguaje documental.

El proceso organización presupone que en ambas formas documentales ocurre el registro de aquello que Dahlberg (1993, p. 214) denomina de "conocimiento en acción", movido por un consenso social que permite que la organización y la representación sean reconocidas, aprendidas. Tal reconocimiento es un factor decisivo para la generación de un nuevo conocimiento.

Como hemos discutido, la concepción social, materializada y cíclica del conocimiento comprende enfoques envueltos en el proceso organización a partir de la interrelación de diferentes conceptos auxiliares - como documento, información y conocimiento (6) - cada cual con su particularidad.

De modo general, el documento remite al estado valorativo atribuido al objeto a partir de los datos - signos lingüísticos registrados, materializados- que, en la condición de entes acabados y autónomos (objetivos), posibilitan la captación mediante los sentidos. La información sería un ente ideal secundario, inacabado, noautónomo, que deriva de los objetos materiales y se caracterizaría por no ser captada por los sentidos. Mientras que el conocimiento seria un ente ideal que tiene como fuente/insumo la información, dependiendo, para eso, de un ente conocedor.

La palabra información deriva de la expresión latina informare - "dar forma"- y puede ser comprendida a partir del proceso de síntesis que compone el propio fenómeno. Esa síntesis congrega elementos subjetivos y objetivos. Los elementos subjetivos se derivan de la interpretación para extraer referentes y sentidos ideales a partir de símbolos, posibilitando procesar, organizar y estructurar datos, dándoles forma. Los datos representan los elementos objetivos - por no depender de los sujetos y porque pueden ser diferenciados de otros datos. Ellos poseen una estructura de naturaleza biológica y psicológica que permite su organización objetiva y no-arbitraria. Tal objetividad atribuida a la organización deriva de su figuración en un contexto histórico y cultural —o sea, que para cada sociedad o cultura podrán valer criterios distintos de organización, cada cual con su objetividad propia. La arbitrariedad puede ocurrir cuando exista la imposición de una forma particular de organización a una sociedad o cultura que no comparte las bases epistémicas impuestas (7).

El conocimiento transciende a la organización y a la estructuración de símbolos. El fenómeno puede depender de la compleja relación entre memorización, análisis, síntesis, visión dialéctica, elaboración de referenciales, evaluación y asimilación, sumándose a la fantasía, imaginación y creatividad de cada sujeto cognoscente. El conocimiento puede llevar al sujeto a asimilar, integrar y reorganizar estructuras, permitiendo la resolución de problemas y la interacción con el entorno. Con el conocimiento es posible crear y recrear sentidos, construir y reconstruir ideas, formar y reformar juicios, producir y reproducir teorías, fundamentar y refundamentar discursos, elaborar y reelaborar visiones de mundo.

La línea de raciocinio citada -que diferencia documento, información y conocimiento- fue desarrollada por Rendón Rojas (2005, pp. 5254) y llevó al autor a defender que información y conocimiento son entes ideales que no pueden ser concebidos como etapas de un mismo proceso. Por ese motivo, el autor establece una jerarquía, por grado de amplitud, entre ambos conceptos. Mientras conocimiento sería un fenómeno exclusivamente subjetivo y más amplio, información seria más restrictivo, manifestándose, de modo objetivado, fuera del sujeto. Ese comportamiento de la información permitiría su inscripción/registro en un soporte, el cual, al ser valorado como documento, traerá datos de in- 
terese social pasibles de organización en una institución informativo-documental.

El carácter de mayor alcance atribuido al conocimiento, cuando es pensado asociado al proceso/objeto de estudio que en él incide organización-, proporciona importantes apoyos para justificar la propia designación de la disciplina OC, cuya actuación se orienta al estudio del amplio espectro de fenómenos observados en el ámbito practico y traducidos racionalmente al ámbito teórico y metodológico.

\section{Consideraciones finales}

El énfasis en el proceso como principal objeto de la disciplina OC clarifica su posición en el campo de la $\mathrm{Cl}$. Partiendo del proceso organización, se podrán observar, además, otros procesos derivados que remiten a otras disciplinas como el Análisis Documental, la Indización, la Clasificación, la Catalogación, la Elaboración de Resúmenes, etc.- en las que se observa la articulación entre conceptos auxiliares.

Así, el objeto de la OC se construye a partir de su relación directa con el concepto conocimiento, que es comprendido como una dimensión amplia que circunscribe los conceptos información y documento. El conocimiento es un ente subjetivo ideal que, objetivado en información, en su exterioridad al sujeto, puede ser registrado en el documento, que a su vez se constituye de datos (contenidos) susceptibles de ser organizados y recuperados $y$, finalmente, aprovechados para el uso. En otras palabras, el conocimiento puede pensarse como un ente formado por un conjunto complejo de informaciones que pueden ser objetivadas en un suporte para darle la materialidad que posibilitará toda la practicidad documental conocida - permanencia en el tiempo, manejabilidad, posibilidad de organización, etc. Para el proceso de organización, el valor documental del objeto presupone la condición sine qua non para la socialización del conocimiento registrado.

A la luz de la importancia atribuida por la sociedad contemporánea -a veces denominada Sociedad de la Información o del Conocimiento- - a la organización para la socialización de la información y el conocimiento, es posible comprender el impulso histórico que motivó el desarrollo del cuadro teórico-metodológico que culminó en la proposición disciplinar de la OC.

\section{Notas}

(1) Sobre esa simultaneidad, la cita de González de Gómez e Orrico (2006, p.14) es esclarecedora: "La producción de conocimiento 'disciplinar' adquiere vitalidad en un movimiento de cierre y abertura: si no tuviesen sus mo- mentos de delimitación y focalización de un dominio movimiento de cierre- los conocimientos se quedarían 'fluidos', 'vagos'; pero si no mantuviesen ese su objeto como algo 'quitado o construido por procesos específicos', dentro de una red de relaciones solidarias con otros objetos, tratados por otras abordajes disciplinares, dentro de todas las redes que ligan el dominio con el universo del cual forman parte - movimiento de abertura-, correrían los riesgos de 'cosificación' de ese objeto, que pasaría a ser naturalizado como cosa-en-sí- y, por tanto, sujeto a su permanente repetición y copia."

(2) Al preguntarse si el libro sería un objeto de la cultura o una simple mercancía de la industria editorial, GarcíaMoreno (2002, p. 229) argumenta que "[...] la cultura y la industria editorial no son incompatibles [...] Sin la industria editorial, el libro y la cultura que representa estaría limitado a una elite, como lo estuvo hasta la aparición de la imprenta, que fue, al final de cuentas, lo que permitió el surgimiento de la producción industrial de libros y su comercialización masiva."

(3) El lenguaje documental es la designación dada al instrumento de indización de un sistema de recuperación de la información, cuya función consiste en propiciar acceso a las informaciones registradas y organizadas en el sistema. El lenguaje documental hace viable el enlace entre el lenguaje del usuario (lenguaje natural) y el lenguaje del sistema (lenguaje artificial).

(4) Conforme explica Hjørland (2003), el estudio del proceso organización ha enfocado el "[...] conocimiento documentado producido por seres-humanos en tipos de documento de uso potencial para otros seres-humanos."

(5) Los autores Esteban Navarro y García Marco (1995, p. 147) reforzaron esa dimensión social, materializada y cíclica del conocimiento, al afirmar que la $\mathrm{OC}$ es una "ciencia tridimensional", puesto que se ocupa de los principios, métodos e instrumentos utilizados para la gestión del conocimiento, bajo un perspectiva tríplice: su representación, su organización y su comunicación documental.

(6) El estudio de tales conceptos puede contribuir a la fundamentación teórica del proceso de organización. Específicamente para este proceso es necesario que se tome en consideración: a) el perfil de los usuarios del sistema; b) la elección del contenido documental a ser indizado; c) el nivel de especificidad/profundidad de indización requerida por el sistema; d) la aceptabilidad y confiabilidad de los productores de los documentos (autores, editores, etc.) contenidos en el sistema; y e) la elección de la mejor forma de representación documental (Jaenecke, 1994).

(7) La arbitrariedad puede ser observada, aún, en el proceso clasificación. En este proceso, precedente inmanente al propio proceso organización, la arbitrariedad ocurre con la falsa sensación de que es posible llegar a la esencia irreductible del ser u objeto clasificado. Según Pombo (1988), toda representación, por medio de una clasificación, reposa en el nivel de la apariencia del ser o del objeto representado. Delante de la existencia de características imperceptibles podrán ocurrir, con relación de un género con otro, especies intermediarias que no fueron explicitadas y que, por eso, serán ignoradas durante el proceso.

\section{Agradecimientos}

Agradezco a Tatiane Pacanaro Trinca y a Miguel Ángel Márdero Arellano por las críticas, sugerencias y traducción que contribuyeron a la redacción de este artículo 


\section{Referencias}

Anderson, J. D. (1995). Organization of knowledge. // Feather, J.; Sturges, P. (eds.) International encyclopedia of information and library science. London: Routledge, 1996. 336-353.

Bachelard, G. (1972). Conhecimento comum e conhecimento científico. // Tempo Brasileiro. 28 (enero - jun. 1972) 47-56.

Bachelard, G. (1996a). A formação do espírito científico: contribuição para uma psicanálise do conhecimento. Rio de Janeiro: Contraponto, 1996a.

Bachelard, G. (1996b). O novo espírito científico. Lisboa: Edições 70, 1996b.

Barité, M. (2001). Organización del Conocimiento: un nuevo marco teórico-conceptual en Bibliotecología y Documentación. // Carrara, K. (eds.) Educação, universidade e pesquisa. Marília, 2001. 35-50.

Beghtol, C. (1998). Knowledge domains: multidisciplinarity and bibliographic classification systems. // Knowl. Org. 25:1/2 (1998) 1-12

Burke, P. (2003). Uma história social do conhecimento: de Gutenberg a Diderot. Rio de Janeiro: Jorge Zahar, 2003.

Campos, M. L. A. (2001). Linguagem documentária: teoria que fundamentam sua elaboração. Niterói: EdUFF, 2001.

Currás, E. (1995). Concierto y desconcierto en la organización del conocimiento actual y su intersección con el mundo de la información. // Scire. 1:1 (en.-jun.1995) 328.

Dahlberg, I. (1993). Knowledg Organization: its scope and possibilities. Know. Org. 20:4 (1993) 211-222.

Dahlberg, I. (1995). Current Trends in Knowledge Organization. // García Marco, F. J. (ed.) Organización del Conocimiento en sistemas de información y documentación. Zaragoza: ISKO - España, 1995. 7-25.

Esteban Navarro, M. A.; García Marco, F. J. (1995). Las primeras jornadas sobre organización del conocimiento: organización del conocimiento e información científica. // Scire. 1:1 (enero-jun. 1995) 149-157.

Esteban Navarro, M. A. (1995). Los lenguajes documentales ante el paso de la organización de la realidad y el saber a la organización del conocimiento. // Scire. 1:2 (1995) 43-71.

Fayet-Scribe, S. (2001). Histoire de la documentation en France: culture, science et technologie de l'information: 1895-1937. Paris: CNRS, 2001.

Freire-maia, N. (1997). A ciência por dentro. 4. ed. Petrópolis: Vozes, 1997.

Fujita, M. S. L. (2001). Organização do conhecimento: algumas considerações para o tratamento temático da informação. // Carrara, K. (eds.) Educação, universidade e pesquisa. Marília: UNESP, 2001. 29-34.

García-Moreno, P. C. (2002) ¿El libro: cultura o industria editorial? // Ribot García, L. A. (eds.). El libro, las bibliotecas y los archivos en España: a comienzos del Tercer Milenio. Madrid: Sociedad Estatal; España Nuevo Milenio, 2002. 227-235.

González de Gómez, M. N. (2003). O contrato social da pesquisa: em busca de uma nova equação entre a autonomia epistêmica e autonomia política. // DataGramaZero. 4:1 (fev. 2003).

González de Gómez, M. N.; Orrico, E. G. D. (2006). Interdisciplinaridade: questões norteadoras e possíveis caminhos. // González de Gómez, M. N.; Orrico, E. G. D. (eds.) Políticas de memória e informação: reflexos na organização do conhecimento. Natal: EDUFRN, 2006. 927.

Guimarães, J.A.C. (2008). A dimensão teórica do tratamento temático da informação e suas interlocuções com o universo científico da International Society for Knwoledge Organization (ISKO). // Revista Ibero-americana de Ciência da Informação. 1 (2008) 77-99.

Hjorland, B. (2003). Fundaments of Knowledge Organization. // Know. Org. 30:2 (2003) 87-111.

Hjorland, B. (1994) Nine principles of Knowledge Organization. // Advances in Knowledge Organization. 4 (1994) 91-100.

Jaenecke, P. (1994) To What end Knowledge Organization. // Know. Org. 21:1 (1994) 3-11.

Japiassu, H. (1977). Introdução ao pensamento epistemológico. 2.nd ed. Rio de Janeiro: Francisco Alves, 1977.

Kuhn, T. S. (2005), A estrutura das revoluções científicas, 9. nd ed. São Paulo: Perspectivas, 2005.

Melo, A. (2006). A construção do objeto turístico: diálogos com a epistemologia de Gaston Bachelard e Pierre Bourdieu. // Seminário de Pesquisa em Turismo do Mercosul - SeminTUR 4 (Caxias do Sul, 7-8 jul. 2006). Caxias do Sul: UCS, 2006.

Otlet, P. (1934). Traité de documentation: le livre sur le livre: théorie et pratique. Bruxelles: Mundaneum, 1934. 372431.

Pombo, O. (1988). Da classificação dos seres à classificação dos saberes. // Revista da Biblioteca Nacional de Lisboa. 2 (1988) 19-33.

Pombo, O. (2003). Epistemologia da interdisciplinaridade. // Seminário Internacional Interdisciplinaridade, Humanismo, Universidade. (Porto, 2003). Porto: Universidade do Porto, 2003 1-18.

Rabello, R.; Guimarães, J. A. C. (2006), A relação conceitual entre conhecimento e documento no contexto da Organização do Conhecimento: elementos para uma reflexão. // Anales del Enancib: a dimensão epistemológica da Ciência da Informação e suas interfaces técnicas, políticas e institucionais nos processos de produção, acesso e disseminação da informação. Marília: FAPESP/Enancib, 2006

Rabello, R. (2009). A face oculta do documento: tradição e inovação no limiar da Ciência da Informação, Tesís Doctoral en Ciência de la Información, Marília, UNESP.: http://www.dominiopublico.gov.br/pesquisa/DetalheObra Form.do?select action $=\&$ co obra $=137497 \quad(2010-03$ 05).

Rabello, R. (2011). A dimensão categórica do documento na Ciência da Informação. // Enc. Bibli: R. Eletr. Bibliotecon. Ci. Inf. 16:31 (2011) 131-156. http://www.periodicos. ufsc.br/index.php/eb/article/view/1518-2924.2011v16n31 p131 (2011-06-20).

Rendón Rojas, M. Á. (2005), Bases teóricas y filosóficas de la Bibliotecología, 2.ed., México-DF: UNAM, CUIB.

Rendón Rojas, M. Á. (2005). Relación entre los conceptos: información, conocimiento y valor. Semejanzas y diferencias. // Ci. Inf. 34:2 (maio/ago. 2005) 52-61.

Smiraglia, R. P. (2002). The progress of theory in knowledge organization. // Library Trends. 50:3, (2002) 330-349.

Vuillemin, J. (1987). Conceito. // Enciclopédia Einaudi. Lisboa: Imprensa Nacional; Casa da Moeda, 1987. v. 37. 77-124.

Woledge, G. (1983). "Bibliography' and "Documentation": words and ideas. // Journal of Documentation. 39:4 (1983) 266-279. 
